\title{
Apoptosis in squamous cell carcinoma of the lung: correlation with survival and clinicopathological features
}

M Ghosh, J Crocker, A Morris

\begin{abstract}
Aims-Apoptosis is recognised as a physiological mechanism for controlling cell numbers and its subversion is thought to contribute to carcinogenesis. The aims of this study were to measure the apoptotic index (AI) in a series of squamous cell carcinomas (SCCs) of the lung using standard histological staining and confirm this by immunohistology using an antibody to an apoptosis specific protein (ASP), and to seek to correlate the AI with clinicopathological parameters.

Methods-Sections of 134 SCCs were stained by haematoxylin and eosin (H\&E) for counting apoptotic bodies to determine the AI (number of apoptotic bodies/ 10000 tumour cells); 26 of these were also stained with anti-ASP antibody and the proportion of ASP positive cells counted. Clinical data were obtained from hospital notes.
\end{abstract}

Results-The mean AI obtained by H\&E staining of all 134 SCCs was 30.3 (SD, 24.75). Anti-ASP staining allowed easy identification of apoptotic bodies, and generated a somewhat higher index (mean, 51.4; SD 39); this was not a result of the selection of tumours because the AI by $H \& E$ in the subset stained with antiASP was 31.1. Regression analysis showed that the correlation between the two values of AI was highly significant $(R s=0.9760 ; p<0.001)$, indicating that the two methods were both reliable measures of apoptosis but that the anti-ASP staining is the more sensitive method. The tumours were grouped into high $\mathrm{AI}(>50)$ and low AI $(<50)$ and survival analysis was carried out. The mean survival of the high AI group was 109 weeks and of the low AI group 72 weeks $(p=0.036)$. Conclusions-Anti-ASP staining is a reliable, easy, and sensitive method for assessing apoptosis in tumour sections and confirms the validity of the AI obtained by $\mathrm{H} \& \mathrm{E}$ staining. AI is a guide to the behaviour of SCCs of the lung. (F Clin Pathol 2001;54:111-115)

Biological Sciences, University of Warwick, Coventry CV4 7AL,

UK

A Morris

Correspondence to: Dr Morris

AMorris@bio.warwick.ac.uk

Accepted for publication 21 August 2000 important in the pathogenesis of Several of the oncogenes and anti-oncogenes that are commonly dysregulated in cancer are involved in the control of apoptosis. Loss of the normal function of these genes, which renders the cell resistant to apoptosis, is felt to be an important step towards the development of neoplasia. Apoptosis continues to take place spontaneously in tumours (as witnessed by its presence in histological sections) and is enhanced by most forms of anticancer treatment such as chemotherapy, radiotherapy, and hormone treatment. ${ }^{1}$

Theoretically, the rate of cell death in a tumour could balance the rate of cell proliferation and thus determine the rate of tumour expansion and therefore the outcome in an individual case. Thus, one would expect tumours with a high apoptotic rate to behave less aggressively and for patients with such tumours to survive longer than those whose tumours have a low apoptotic rate. However, in practice this is often not the case..$^{2-4}$

The estimation of apoptosis in histological sections is a long and arduous process. Apoptosis occurs very infrequently in most tumours ( $<1 \%$ of cells) and therefore large numbers of cells need to be counted systematically for the results to be accurate and reproducible. It is easy to mistake lymphocytes and mitotic bodies for the dark pyknotic nuclei of apoptotic cells, particularly at low magnification. To ease the process of identification and avoid these mistakes, staining methods have been devised that identify the strand breaks of DNA in apoptotic cells (in situ end labelling (ISEL) ${ }^{5}$ and in situ nick translation $\left(\right.$ TUNEL) ${ }^{6}$ ). Yet another method of singling out apoptotic cells in a section is to stain immunohistochemically for apoptosis specific protein (ASP). This protein, which bears a serendipitous similarity to the oncogene c-Jun, is a modified cytoskeletal protein that is expressed in apoptotic cells from very early to very late stages, but not in necrotic or viable cells. ${ }^{7}$ It can be detected immunologically with an antibody raised against amino acids 73-87 of the human c-Jun protein (anti-c-Jun/AP-1 (Ab-2)). This antibody has been used to detect and quantify apoptotic cells in histological sections of Burkitt's lymphoma and was found to be more sensitive than the ISEL or TUNEL techniques. ${ }^{8}$

We enumerated apoptotic cells using haematoxylin and eosin (H\&E) staining in 134 cases of squamous cell carcinoma of the lung. In some of these cases we also used the ASP antibody to see whether the two methods correlated. Our aim was to determine whether the apoptotic indices (AIs) in the sections correlated with survival or any other clinicopathological marker. 


\section{Materials and methods}

PATIENTS AND TUMOUR MATERIAL

The archival material used for our investigations consisted of paraffin wax blocks containing tumours resected from 134 patients with squamous cell carcinoma (SCC) of the lung in 1977. These cases were reported in 1991 by Boldy et al, who investigated interphase argyrophilic nucleolar organiser region (AgNOR) counts and DNA ploidy of these tumours. ${ }^{9}$ The patients ranged in age from 40 to 73 years (mean, 60.6 ) and $89.5 \%$ (120 of 134) were men. Seventeen patients died in the first 30 days after surgery. Follow up was for 10 years and was complete, apart from one patient who was lost to follow up after 110 months; 33 $(27 \%)$ patients were alive at five years and 23 $(17 \%)$ were alive at 10 years.

ESTIMATING APOPTOSIS IN H\&E SECTIONS Paraffin wax sections were stained with $\mathrm{H} \& \mathrm{E}$ using a standard technique and examined through an oil immersion lens $(\times 630)$. Apoptotic cells were identified by the following criteria: (1) cells showing pronounced condensation of chromatin and cytoplasm; (2) cytoplasmic fragments containing condensed chromatin; and (3) intracellular and extracellular chromatin fragments. The eosinophilia of the cytoplasm of apoptotic cells was not a useful criterion because the cytoplasm of SCCs of the lungs tends to be eosinophilic.

Apoptosis is a rare event in tissues $(<1 \%$ in tumour sections) and there is a tendency for apoptotic bodies to cluster in adjacent fields. Thus, if only a few fields are examined, the count can vary widely from one examination to another. This variation can be eliminated considerably by increasing the size of the sample. As more and more cells are counted the variation decreases sharply and then plateaus off until counting more cells does not improve the results greatly. The "running mean" method can be used to determine the minimum number of cells to be counted for the results to be reliable. This method consists of calculating stepwise an AI (number of apoptotic cells/100 malignant cells) for every 100 malignant cells until the difference between the successive means becomes negligibly small $(<1 \%$ of the index). In the lung cancer slides this occurred after counting 1500 cells. We found that examining 10 fields under the oil immersion lens was always enough to include 1500 cells.

Ten fields were chosen from different parts of the tumour section avoiding areas of normal epithelium, fibrous stroma, and necrosis. The numbers of apoptotic cells and the numbers of malignant cells in each field were recorded. Apoptotic bodies occurring in distinct groups and likely to be originating from the same cell were recorded as one apoptotic cell. The count in all 10 fields were added and expressed as the AI (number of apoptotic bodies/10 000 malignant cells).

To check whether the results were reproducible, each slide was counted again on a second occasion six months later while being "blinded" to the initial result. Ten fields were counted using the same protocol as before. On
Spearman's rank correlation testing the sets of apoptotic indices obtained on the two separate occasions were found to correlate very closely $(R \mathrm{~s}=0.9984 ; \mathrm{p}<0.001)$.

\section{STAINING WITH ANTIBODY TO ASP}

Twenty six tumours were chosen at random for the determination of the AI using an anti-ASP antibody. The antibody used was anti-c-Jun/ AP-1 (Ab-2), obtained from Oncogene Science (Cambridge, UK). Dewaxed and hydrated histological sections were microwaved (750 W, 15 minutes) and were immunoperoxidase stained using the Vectastain Elite kit and DAB substrate kit for peroxidase (Vector Laboratories Inc, Peterborough, UK), counterstaining with Mayer's haemallum. Sections of a lymph node were used as positive controls. For negative controls sections from the same tumours were used, omitting the primary antibody from the staining protocol.

Using this technique, apoptotic cells displayed brown cytoplasmic staining in addition to the dark, condensed (and in many cases fragmented) nucleus. Remains of apoptotic cells within phagosomes also took on a brown stain. These apoptotic cells stood out starkly among the malignant cells, which had only taken up the light blue counterstain of Mayer's haemallum.

Apoptosis was quantified using the same protocol as for H\&E. In brief, 10 oil immersion fields were chosen at random and the numbers of apoptotic and malignant cells in each field were counted. The slides were counted while being blinded to the result of the AI obtained from the corresponding $\mathrm{H} \& \mathrm{E}$ stained sections. The final AI was expressed as apoptotic cells/10 000 malignant cells.

PLOIDY AND AgNOR DETERMINATIONS

AgNORs were enumerated as in the study of Boldy and colleagues ${ }^{9}$; namely, $3 \mu \mathrm{m}$ paraffin wax sections were cut, dewaxed, then stained by the standard one step silver colloid technique. The numbers of cells to be used in the counting procedure were arrived at by the wandering mean method. This gave a number of 50 cells. All black dots were counted in each cell.

DNA flow cytometry was performed on disaggregated cells, stained by propidium iodide. At least 30000 events were counted. The G0/G1 peak coefficient of variation was calculated electronically. A specimen was considered aneuploid if a peak was seen, separate to the main peak for normal cells.

\section{STATISTICAL ANALYSIS}

For survival analysis, survival to death of recurrent disease was used as follow up variable, censoring patients dying of other causes. Kaplan Meijer curves were plotted, and differences in survival were analysed with the log rank test. Grouping was done according to the optimal $p$ value. Multivariate analysis was done with Cox regression, entering only the variables that were significant in univariate analysis as discrete variables at the optimal cut off value (enter limit, 0.5; remove limit, 0.1 ). 


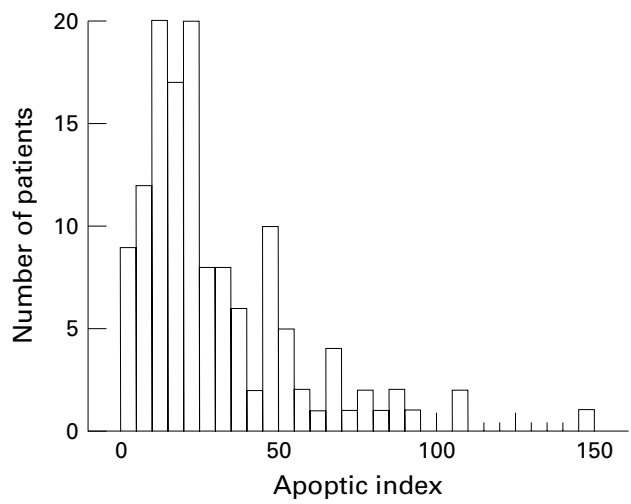

Figure 1 Frequency distribution of apoptotic indices of 134 squamous cell lung carcinomas determined by haematoxylin and eosin staining.

Table 1 Comparison of apoptotic indices (AIs) obtained by haematoxylin and eosin (HEE) staining and staining with an antibody to apoptosis specific protein (ASP)

\begin{tabular}{lrclll}
\hline & Min. & Max. & Mean & Median & SD \\
\hline AI (H\&E) & 9.2 & 90.6 & 31.1 & 20.6 & 22.9 \\
AI (ASP) & 13.9 & 159 & 51.4 & 32.8 & 39 \\
\hline
\end{tabular}

AI, number of apoptotic bodies/10 000 malignant cells.

These tests were done with SPSS. Other analyses were carried out with Astute.

\section{Results}

AI OF LUNG CANCERS

The value of the AIs obtained by H\&E staining for all 134 cases ranged from 2.4/10 000 malignant cells to $145.5 / 10000$ malignant cells, with a mean of 30.29 (SD, 24.75; median, 22.3).

Figure 1 plots the frequency distribution of the AIs of these tumours. It can be seen that the distribution is asymmetric with a suggestion of bimodality. It is clear from the graph that most cases have a relatively low rate of apoptosisbetween 10-25/10 000 malignant cells, with a peak at about 20 . There is a second peak at 50 beyond which the numbers dwindle rapidly.

In those cases where ASP staining was used to identify apoptotic cells, the counts obtained were higher than the corresponding $\mathrm{H} \& \mathrm{E}$ derived counts. Table 1 shows a comparison of the AIs in the 26 cases where both staining methods were used. Using Spearman's rank

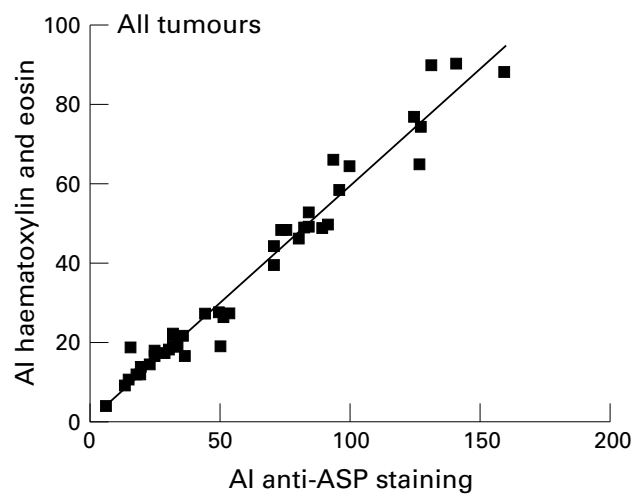

Figure 2 Correlation between apoptotic indices (AI) as determined by haematoxylin and eosin staining and anti-apoptosis specific protein (ASP) staining.

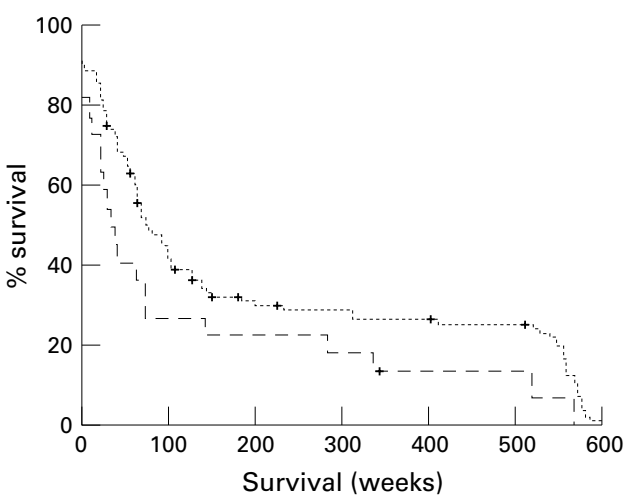

Figure 3 Survival curves for patients with low (upper curve) and high apoptotic indices.

correlation test (fig 2) the two sets of AIs correlated very strongly $\quad(R \mathrm{~s}=0.9839$; $\mathrm{p}<0.001)$.

In all cases the count obtained by anti-ASP staining was higher than that obtained by H\&E staining. This was not unexpected because ASP staining had clearly picked up cells in the very early and very late stages of apoptosis, which would not have been identified by their morphological features alone. What is important for our purposes is that the two sets of values correlated so very strongly. This indicates that compared with ASP, H\&E underestimated apoptosis by an almost fixed proportion in every case. Thus, although the absolute value of apoptosis obtained by $\mathrm{H} \& \mathrm{E}$ would certainly be an underestimate of the "real" value, the indices are still valid for comparing the apoptotic states of different tumours.

CORRELATION BETWEEN AI AND SURVIVAL

Patients were grouped into high and low apoptosis groups on the basis of the AI of the tumour: > 50 was regarded as high. Figure 3 shows the survival curves for patients with low and high AI. The mean survival of patients was 172 and 109 weeks, respectively $(p=0.036)$. In multivariate survival analysis, AI appeared to have additional prognostic value to stage.

\section{CORRELATION BETWEEN AI AND OTHER}

PATHOLOGICAL MARKERS

There was no significant difference in AI between patients in different disease stages. No significant difference in AI was found in

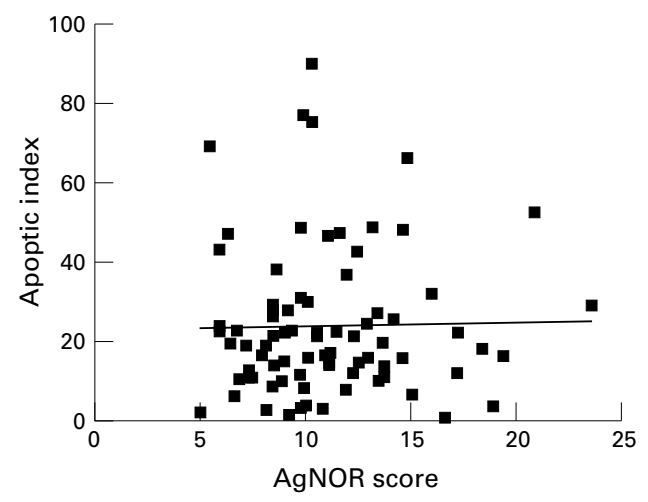

Figure 4 Correlation between apoptotic indices and interphase argyrophilic nucleolar organiser region (AgNOR) counts in squamous cell carcinoma. 
Table 2 Survival times of patients in each AgNOR (interphase argyrophilic nucleolar organiser region) group and correlation between apoptotic index and survival within each group

\begin{tabular}{|c|c|c|c|c|c|c|c|c|}
\hline Group & $n$ & Min. & Max. & Mean & $S D$ & Median & $R s$ & p Value \\
\hline I & 14 & 5 & 134 & 50.6 & 54.7 & 15.5 & 0.1011 & 0.736 \\
\hline II & 37 & 4 & 135 & 36 & 41.4 & 17 & -0.0065 & 0.966 \\
\hline III & 21 & 3 & 133 & 35.3 & 46.9 & 16 & -0.0951 & 0.678 \\
\hline IV & 9 & 5 & 132 & 52.7 & 57.3 & 19 & 0.1333 & $>0.05$ \\
\hline
\end{tabular}

Group I AgNOR < 8. Group IIAgNOR 8-12. Group III AgNOR 12-16. Group IV AgNOR $>16$

patients with different degrees of lymphatic spread (N0, N1, N2), different grades of histological differentiation (well, moderate, and poorly differentiated), or differences in ploidy (diploid and aneuploid).

Boldy et al had obtained AgNOR counts (a measure of cell proliferation) for 104 of the present series of patients, which they described in their paper. ${ }^{9}$ The counts ranged from 4.6 to 24/cell nucleus/specimen. The mean AgNOR count was 11.3 (SD, 3.9). Using Spearman's rank correlation test, no correlation was found between the AgNOR counts and the corresponding AIs ( $\mathrm{s} s=0.1070 ; \mathrm{p}=0.280$; fig 4 ).

For the purposes of analysis, Boldy et al had divided the tumours into four groups according to the AgNOR counts, namely:

- Group I AgNOR $<8$.

- Group IIAgNOR 8-12.

- Group III AgNOR 12-16.

- Group IV AgNOR > 16.

We looked for a correlation between AI and survival between each of these four groups. Table 2 describes the survival times in each group. The last two columns describe the coefficients of correlation (Spearman's rank correlation) between $\mathrm{AI}$ and survival.

\section{Discussion}

Even without anticancer treatment, tumour cells undergo apoptosis for several reasons. Direct attack by cytotoxic $\mathrm{T}$ cells and tumour necrosis factor $\alpha$ (TNF- $\alpha)$ released by infiltrating macrophages are two examples of triggers. Mild hypoxia not strong enough to cause necrosis can also result in apoptosis, as can genetic aberrations within cancer cells, which act as an internal trigger. ${ }^{1}$ It would be expected that patients with tumours that display a higher rate of apoptosis would survive longer. In our series of SCCs of the lung the opposite was observed, with patients whose tumours showed a low AI surviving longer. Stammler and Volm in their 178 cases of non-small cell lung carcinoma also described a significant trend towards shorter survival in high AI tumours. ${ }^{2}$ Komaki et al found no correlation between apoptosis and survival in 173 cases of resected non-small cell lung carcinoma, but when a subgroup of adenocarcinomas and large cell lung carcinomas were considered separately, high apoptosis was associated with a significant chance of metastasis at five years. ${ }^{3}$ Tormanen et al in their 75 cases of non-small cell lung carcinoma found a significantly shorter survival time in patients with high AIs. ${ }^{10}$

High apoptosis has also been found to be associated with a significantly shorter survival in carcinoma of the cervix, ${ }^{4}$ transitional cell bladder carcinoma, ${ }^{11}$ and breast cancer. ${ }^{12}$ Other studies investigating gastric cancer ${ }^{13}$ and ovarian cancer $^{14}$ found no correlation between apoptosis and survival. In a series of 91 cases of breast cancer, Shao and co-workers found a high apoptotic rate to be associated with better prognosis. $^{15}$

Overall, it is fair to comment that in most cancers, including lung cancer, apoptosis does not have the effect on survival that would be expected on theoretical grounds; that is, high apoptosis does not predict better prognosis. There are several possible explanations.

First, it may be that what is being measured as apoptosis in histological sections is inaccurate and therefore the conclusions derived from such measurements are likely to be false. There have always been serious doubts about the rationality of measuring a dynamic process such as apoptosis in histological sections. The amount of apoptosis observed in a tissue at any given time is a result of the balance between the speed of apoptotic body formation and the speed of phagocytosis, both of which are known to vary independently. The only way to estimate this accurately is in a dynamic system-for example, tissue culture. A histological section in effect provides data concerning apoptotic state rather than apoptotic rate.

The second possibility is that increased apoptosis could be a marker of tumour progression. It could also merely be an epiphenomenon, a sign of rapid tumour growth outstripping the rate of new vessel formation resulting in mild hypoxia and apoptosis. In either case, one would expect higher AIs to be found in tumours that are larger, less well differentiated, aneuploid, and with more metastatic potential. None of these correlations was found in our series of lung cancers. Three other groups have reported a similar lack of correlation in lung tumours. ${ }^{2}{ }^{316}$ Tormanen et al found higher AIs in poorly differentiated lung cancers, but they found no correlation between apoptosis and TNM stage. ${ }^{10}$

In other tumours that have been investigated there seems to be no consistent relation between apoptosis and pathological parameters. An association between high apoptosis and poor histological differentiation has been described in carcinoma of the cervix, ${ }^{17}$ ovary, ${ }^{14}$ and prostate.$^{18}$ In breast cancer, high apoptosis has been described in association with poor differentiation, aneuploidy, loss of oestrogen receptors, and poor prognosis, ${ }^{12}{ }^{19}$ whereas others have reported a significant correlation between high apoptosis and better differentiation $^{15}$ and a lower risk of lymph node metastasis. $^{20}$ In oesophageal ${ }^{21}$ and gastric ${ }^{22}$ carcinoma, high apoptosis is seen more often in well differentiated tumours.

Similarly, although a direct correlation between apoptosis and proliferation is usually seen in many tumours (bladder carcinoma ${ }^{11}$, breast cancer, ${ }^{122324}$ and colonic cancer ${ }^{25}$ ) in lung cancer no such correlation was found either by us or by two other groups (Eerola and colleagues $^{26}$ and Tormanen and colleagues ${ }^{10}$ ). It would seem logical that the combination of a low proliferation rate and high apoptotic rate 
would confer a good prognosis, but we did not find this to be the case.

In analysis of survival there was no difference in outcome between lung tumours with high AgNOR/low AI values, which are expected to accumulate cells much more quickly than those with low AgNOR/high AI values. One could conclude that mere numerical superiority is not as important in tumour progression as the nature of the malignant cells that are being produced. Whether the cells are able to invade locally or metastasise to distant organs is likely to be a key determinant of outcome. Of course, aberrant apoptosis could influence some of these characteristics as well.

The determination of apoptotic rates in sections of tumour material is complicated by the rarity of apoptotic bodies, requiring the counting of large numbers of cells, and by the fact that they tend to cluster. Others have approached this methodological problem by systematic sampling. ${ }^{27}$ We have adopted random sampling, using the running mean method and counting apoptoses in H\&E stained sections, and confirmed the AI so obtained by a second assessment done blind in the same way, and also by a third assessment performed on a subset of the tumours using an immunological method. The three values correlated very closely and so the AI we have obtained has a very high degree of confidence.

We can therefore conclude that the data we present here, together with that of others, clearly indicates that $\mathrm{AI}$ as measured by histological techniques is a guide to prognosis in squamous lung cancer.

The authors acknowledge the generous help of $\mathrm{K}$ Jenner and $\mathrm{J}$ Starczynski in carrying out the immunohistochemistry, without which this work would not have been possible.
van Diest for his help with the survival statistics.

1 Kerr JFR, Winterford CM, Harmon BV. Apoptosis: its significance in cancer and cancer therapy. Cancer 1994;73:2013-26.

2 Stammler G, Volm M. Apoptosis in NSCLC as related to drug resistance and prognosis. Apoptosis 1996;1:95-9.

3 Komaki R, Fujii T, Perkins P, et al. Apoptosis and mitosis as prognostic factors in pathologically staged N1 NSCLC. Int prognostic factors in pathologically stage

4 Levine EL, Renehan A, Gossiel R, et al. Apoptosis, intrinsic radiosensitivity and prediction of radiotherapy response in radiosensitivity and prediction of radiotherapy res
cervical carcinoma. Radiother Oncol 1995;37:1-9.

5 Wijsman JH, Jonker RR, Keijzer R, et al. A new method to detect apoptosis in paraffin sections: in situ end-labelling of fragmented DNA. F Histochem Cytochem 1993;41:7-12

6 Gavrieli Y, Sherman Y, Ben-Sasson SA. Identification of programmed cell death in situ via specific labelling of nuclear DNA fragmentation. F Cell Biol 1992;119:493501
7 Grand RJA, Milner AE, Mustoe T, et al. A novel protein expressed in mammalian cells undergoing apoptosis. Exp Cell Res 1995;218:439-51.

8 Panchalingam S, Reynolds GM, Lammas DA, et al. Simple method for pretreatment of tissue sections for the detection of apoptosis by in situ end labelling and in situ nick translation. 7 Clin Pathol: Mol Pathol 1996;49:M273-7.

9 Boldy DAR, Ayres JG, Crocker J, et al. Interphase nucleolar organiser regions and survival in squamous cell carcinoma of the bronchus: a 10 year follow up study of 138 cases. Thorax 1991;46:871-7.

10 Tormanen U, Eerola A-K, Rainio P, et al. Enhanced apoptosis predicts shortened survival in NSCLC. Cancer Res 1995:55:5595-602.

11 Lipponen PK, Aaltoma S. Apoptosis in bladder cancer as related to standard prognostic factors and prognosis. $\mathcal{F}$ Pathol 1994;173:333-9.

12 Lipponen P, Aaltoma S, Kosma VM, et al. Apoptosis in breast cancer as related to histopathological characteristics and prognosis. Eur F Cancer 1994;30A:2068-73.

13 Koshida Y, Saegusa M, Okayasu I. Apoptosis cell proliferation and expression of bcl2 and bax in gastric carcinomas: imunohistochemical and clinicopathological study. $\mathrm{Br} f$ Cancer 1997;75:367-73.

14 Diebold J, Baretton G, Felchner M, et al. Bcl2 expression, p53 accumulation, and apoptosis in ovarian carcinomas. Am f Clin Pathol 1996;105:341-9.

15 Shao ZM, Jiang M, Wu J, et al. Inhibition of apoptosis in human breast cancer as related to prognosis. Oncol Rep 1996;3:1183-6.

16 Kargi HA, Aktas S, Sagol O, et al. Apoptosis bcl2 and p53 expression and their relation to tumour stage in non-small cell lung carcinomas. Cancer Lett 1997;116:185-9.

17 Shoji Y, Saegusa M, Takano Y, et al. Correlation of apoptosis with tumour cell differentiation, progression, and HPV infection in cervical carcinoma. F Clin Pathol 1996;49:1348.

18 Vesalainen S, Lipponen P, Talja M, et al. Histological grade, perineural infiltration, tumour infiltrating lymphocytes and apoptosis as determinants of long term prognosis in prostatic adenocarcinoma. Eur f Cancer 1994;30A:1797803.

19 Jong JS de, Diest PJ van, Baak JPA. Number of apoptotic cells as a prognostic marker in invasive breast cancer. $\mathrm{Br} \mathcal{F}$ Cancer 2000;82:368-73.

20 Ohashi K, Nemoto T, Eishi Y, et al. Proliferative activity and p53 protein accumulation correlate with early invasive trend and apoptosis correlates with differentiation grade in oesophageal squamous cell carcinomas. Virchows Arch 1997;430:107-15.

21 Shinohara T, Ohshima K, Murayama H, et al. Apoptosis and proliferation in gastric carcinoma - the association with histological type. Histopathology 1996;29:123-9.

22 Sierra A, Castellsague X, Tortola S, et al. Apoptosis loss and bcl2 expression-key determinants of lymph node metastasis in T1 breast cancer. Clin Cancer Res 1996;2:1887-94.

23 Samoszuk MK, Sallash G, Chen K, et al. Association between flow cytometric $S$ phase fraction and apoptotic rate in breast cancer. Cytometry 1996;26:281-5.

24 Frankfurt OS, Robb JA, Sugarbaker EV, et al. Apoptosis in human breast and gastrointestinal carcinomas-detection in histological sections with monoclonal antibody to single stranded DNA. Anticancer Res 1996;16:1979-88.

25 Baretton GV, Diebold J, Christoforis G, et al. Apoptosis and immunohistochemical bcl2 expression in colorectal adenomas and carcinomas. Cancer 1996;77:255-64.

26 Eerola AK, Tormanen U, Rainio P, et al. Apoptosis in operated small cell lung carcinoma is inversely related to tumour necrosis and p53 immunoreactivity. $f$ Pathol 1997 ; 181:172-7.

27 van de Schepop HAM, de Jong JS, van Diest PJ, et al. Counting of apoptotic cells: a methodological study in invasive breast cancer. I Clin Pathol: Mol Pathol 1996;49: M214-17. 